\title{
Semantic Social Networks for Integrated Healthcare
}

\author{
Eleni Kaldoudi and Nikolas Dovrolis \\ School of Medicine, Democritus University of Thrace, \\ 68100 Alexandroupoli, Greece \\ kaldoudi@med.duth.gr, ndovroli@alex.duth.gr
}

\begin{abstract}
An important aspect of integrated care is the provision of personalized patient empowerment and decision support services, especially in case of the chronic patient with comorbidities. The paper discusses a blending of notions of social networks and semantic technologies in order to develop comprehensive personalized and dynamic models of the chronic comorbid patient, their environment and healthcare related issues, procedures, etc., so as to be able to support meaningful patient empowerment and decision support services.
\end{abstract}

Keywords: Social networking, Semantic technologies, Heterogeneous social Networks, UMLS, Healthcare integration, Actor network theory.

\section{Introduction}

The first decades of applications of information technology in medicine have targeted the health care enterprise and services provided therein. A major technological challenge has been the integration of various information systems and services to support the healthcare enterprise with emphasis on the tertiary level (e.g. hospitals). Towards this goal, a number of standards and standard communication protocols have been developed and implemented, with variable, rather questionable, success [1].

Along with this, the notion of integrated care has risen and now is a central issue in the domain of healthcare. Integrated care has many meanings [2]; the notion of "reunite parts of a whole" is an underlying commonality, whether integration refers to integration within different healthcare settings or in terms of integration of healthcare, social care, long-term and self-care or even integration of patient management for different conditions. In any case, a patient-centered bottom-up approach is favored [2],[3]. Closely related is the concept of patient empowerment, which has emerged as a new paradigm that can help improve medical outcomes while lowering costs of treatment by facilitating self-directed behavior change. The concept seems particularly promising in the management of chronic diseases [4],[5] and it is directly connected with personalized patient services and preventive measures. An important aspect of integrated care is the provision of personalized patient empowerment and decision support services, especially in case of the chronic patient with comorbidities. Comorbidity refers to the presence of one or more disorders in addition to a primary disease or disorder (either independently, or as a consequence of the primary condition or otherwise related) [6]. Comorbidity management is a hot topic in current medical literature [7], [8]. When addressing disease in the presence of comorbidities, each 
different medical condition the patient presents should not be viewed independently, but a "patient as a whole" view approach should be followed [9]. This places an emphasis on and extra burden of dealing successfully with all associations, interactions, co-dependencies, implications, adverse events, etc. that occur between different conditions co-presenting at the same patient at the same time, as well as between the different treatment regimens these conditions involve.

The goal of our work is to address integrated care for the chronic patient with comorbidities. Although a lot of work has been conducted towards a common understanding of the healthcare enterprise, even in the special case of the provision of home care for the chronic patient, e.g. [10], an analysis and definition of the personal environment of the healthy citizen and the patient is still missing. In our approach we propose the blending of notions of social networks and semantic technologies in order to develop comprehensive personalized and dynamic models of the chronic comorbid patient, their environment and healthcare related issues, procedures, etc., so as to be able to support meaningful patient empowerment and decision support services. Section 2 of this paper gives a brief overview of the current state of the art in social network technologies highlighting a novel approach adopted by our work. Section 3 discusses semantic tools and technologies in healthcare that can be invoked for building social network relationships. Section 4 presents the proposed model for the semantic social network and some preliminary implementation approaches while a discussion is given in Section 5.

\section{Heterogeneous Social Networks}

A conventional social network approach concentrates on the network of humans, presumably based on some common social or professional interest - any implied artefacts or concepts are of no interest and are not represented or accounted for in such networks. Here the focus is to establish relationships and connections among humans. These are based on some commonly shared interest, object and/or concept; however, this is only implied and not really accounted for in the network. Such an example from the (of the many) in the field of healthcare is the CarePages (http://www.carepages.com/) a social network of people collaborating together to share the challenges, hopes and victories of anyone facing a life-altering health event. Social networking in this sense is good at realizing and representing links between people, but it doesn't explain what connects those particular people together and what connects those and not others [11].

One way to provide meaning to social networks is to establish relationships and promote self-organization into communities based on shared interests, and even more on specific items of interests. Recently the term 'object-centered sociality' was introduced [12] to describe the fact that strong social relationships are built mainly when individuals are grouped together around a shared object that mediates the ties between them. This can be achieved by organizing the network around the content people create together, comment on, link to, annotate similarly etc. [13]. In this case emphasis is placed on the connections between the humans and the objects and social 
interactions are basically established on the basis of commonly shared objects. This new approach to sociality has drawn attention, and current state-of-the-art research in the area involves various ways to exploit object-oriented sociality to the benefit of the community. An indicative example from the field of healthcare is the PatientsLikeMe site (http://www.patientslikeme.com/), which connects people based on their health issues and related shared experiences.

In either case, the focus is on trying to establish connections based on human action, agency and perception. A recent work, introduced the notion of heterogeneous social networks, where humans and social objects are uniformly treated as equal actors [14]. This paradigm follows the view and concepts of the actor-network theory (ANT). The basis of actor-network theory, a sociological theory developed in the 1980's [15], is the concept of the heterogeneous network, that is, a network containing many dissimilar elements, all treated as inseparable. This is the so-called principle of generalized symmetry, whereby human and non-human (e.g. artifacts, organization structures) are assigned equal amounts of agency. The basis of actor-network theory is the concept of the heterogeneous network, that is, a network containing many dissimilar elements, including both human and non-human entities. Using ANT one can show how things are attracted into or excluded from these networks, how some linkages work while others don't, and how connections are strengthened to make themselves stable and durable by linking to other networks and things. Some of these connections link together to form and identifiable entity of assemblage that can exert force - this is referred to as 'actor'.

\section{Semantics for Building Social Network Connections}

In implementing such a network, major challenges include a unified treatment and representation of all types of possible actors as well as the development of a social behavior for various nonhuman actors, and subsequently their own associations and networks. Both challenges can be addressed by concepts and technologies of the Semantic Web. In the conventional Web, a resource can be described via an XHTML/XML document, where various tags are used to annotate the document, mainly regarding its presentation, not conveying any semantics about the resource itself. In order to describe a resource the W3C Resource Description Framework (RDF) [16] is commonly used to represent metadata about a resource in the form of triples: subject, predicate, object. Generally, the subject can be the resource itself while the predicate can be any relationship as defined in any XML namespace published on the Web. The object can be an explicit value, but also a dereferencable URI. This way, an RDF triple can link the description of a resource with other sources of information on the Web, thus creating a worldwide graph-like linking of resources, what is currently termed Linked Open Data (LOD) cloud [17],[18].

The building blocks of the Semantic Web are considered to be ontologies, i.e. formal descriptions of parts of the world [19] that guide the specification and generation of the triplets. There are numerous ontologies that are specific to domain, resource type and objective (also known as application ontologies), but there are only 
a few general ones (known as upper or foundation ontologies) that are used frequently to build the former. One of the basic requirements is a unified and rich description of all actors to form as a basis for their social presence and their interactions within the social environment. This description includes two main aspects. The first is a domain specific description of the profile for both humans and nonhuman entities. Such a social profile can be described with a variety of domain specific schemata or even ontologies. For example, humans' profile can be based on the FOAF (Friend Of A Friend) (http://www.foaf-project.org/) ontology, mainly used to describe people, the links between them and the things they create and do.

In the healthcare domain there is a plentitude of formal controlled vocabularies and ontologies, for example see the NCBO BioPortal [20], [21] for an indicative list. Although a tremendous amount of effort has been devoted by the medical informatics community, a universal standard medical terminology and/or ontology has yet to be achieved [1]. Thus current state of the art presents a wealth of numerous diverse and often overlapping vocabularies, terminologies and ontologies, e.g. more than 340 available only in NCBO Bioportal. The ontologies used include amongst else for prominent medical ontologies such as SNOMED-CT (Systematized Nomenclature of Medicine - Clinical Terms), ICD9/10 (International Statistical Classification Diseases and Related Health Problems), Body System (body system terms used in ICD11), MeSH (Medical Subject Headings), NCI (Meta)Thesaurus, Galen (the high level ontology for the medical domain), HL7 (the Normative RIM model v2), Biomedical Resource Ontology (BRO, a controlled terminology of resources to improve sensitivity and specificity of Web searches).

Research efforts have focused on producing mappings between different terminologies, probably the most notable contribution is the UMLS mapping between more than 100 of the most commonly used controlled vocabularies in healthcare [22]. Indeed, UMLS (The Unified Medical Language System, http://www.nlm.nih.gov/research/umls/) also provides a semantic network of concepts via sets of semantic relationships. This network has 135 semantic types and 54 relationships, and covers with these effectively all concepts and connections in the healthcare domain.

\section{Semantic Social Networks for Integrated Healthcare}

A recent thorough treatment of the domain of chronic comorbid patient [23] suggests that one way to improve care is to cross reference evidence and guidelines for each condition with guidelines on comorbidities. As it has already been argued in literature [23], simple cross referencing of existing medical evidence and guidelines for all possible combinations of conditions would quickly make it unreadable and thus inefficient. Thus, the goal of our work is to follow a personalized and semantically enriched approach to create dynamic cross referencing of semantically related evidence data. The effort lies in semantic interlinking of three types of data (a) medical ground knowledge (b) up-to-date medical evidence and (c) personal patient data in order to create a personalized model of the disease and comorbidities progression pathways and trajectories (Fig. 1). 


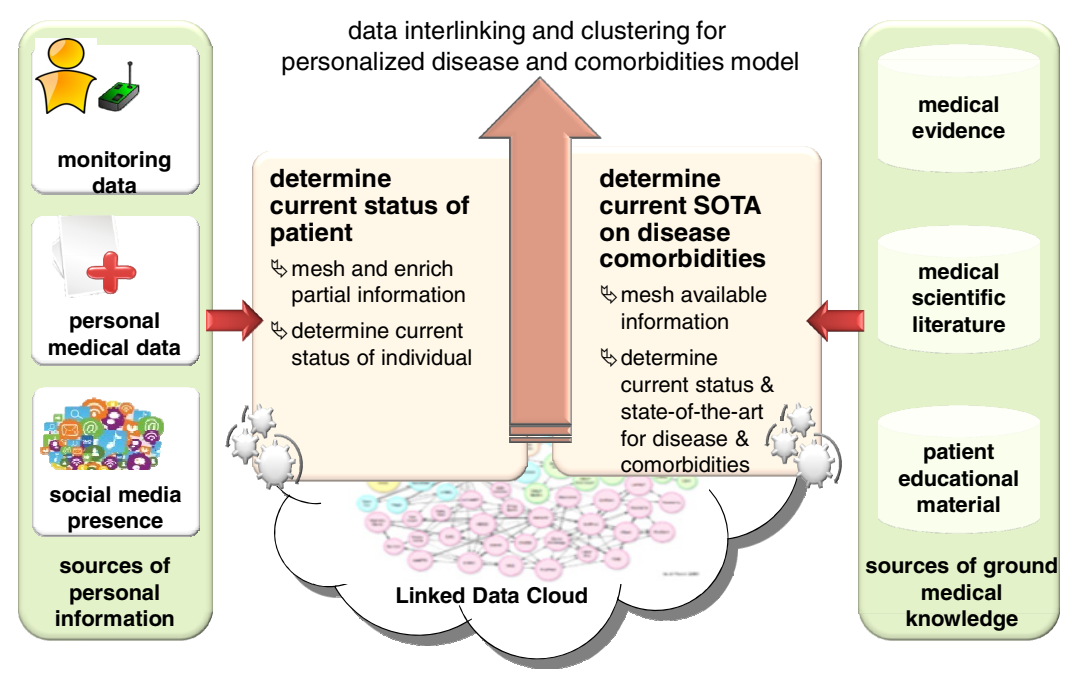

Fig. 1. Shifting focus towards personalized comorbidity management

Following the line of thought presented above, the health environment for the patient and/or the healthy citizen comprises of various coexisting and strongly interlinked entities: (a) individuals, including patients, healthy citizens and healthcare professionals; (b) organizations, including any institutional or organizational entity involved in any way in the healthcare process, e.g. healthcare providers, social services, health insurances, medical research institutions, research projects, pharmaceutical companies, well-being and fitness clubs, etc.; (c) health conditions, i.e. any health or medical condition; and (d) health interventions, including diet, life-style, therapy and drugs, supporting devices, etc. The UMLS semantic network [24] concepts and relationships have been used a basis for developing the proposed model shown (in a simplified, partial view) in Fig. 2.

All entities presented in this model can be viewed as symmetric actors within a heterogeneous social structure which represents the health and healthcare environment of the healthy citizen and patient, thus building and representing all interactions and effects amongst different concepts, objects and humans. A variety of ANT concepts and constructs can then be employed to support development of patient centered services for integrated care like symmetry, translation, black boxes, immutable mobiles and obligatory points of passage.

Symmetry: all things (humans and non-humans, concepts or real world) are treated in the same way, and are assumed to be capable of exerting force and joining together, changing and being changed by each other.

Translation: what happens when human and nonhuman entities come together and connect, changing one another to form links. At each such connection, one entity is working upon another to translate or change it to become part of a network of coordinated things and actions. In our approach, the concept of translation can be used to enhance inherently weak health relationships (such as a "may_cause disease") via multiple connections especially based on evidence based medicine findings and published research papers (e.g. systematic reviews and meta-analyses). 


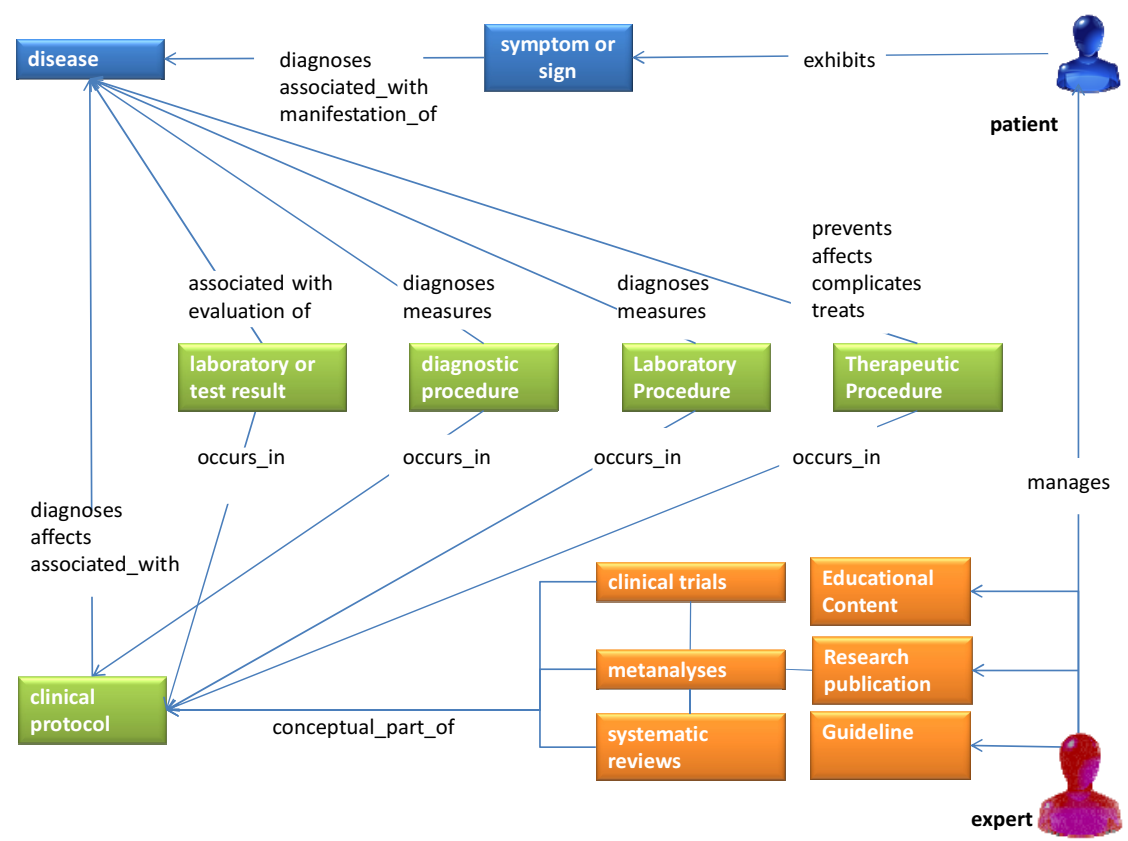

Fig. 2. A simplified partial view of the proposed model which is based on the UMLS semantic network

Black Boxes: Dynamics among actors to translate each other can become stabilized and the network can settle into a stable process or object that maintains itself. This can be viewed as a black-box, concealing all negotiations that brought it into existence. In our approach, a chronic condition (together with all its inflicting factors, treatments, monitoring, existing co-morbidities, etc.) well established in a patient could be treated as a black-box.

Immutable Mobiles: these are only visible within a particular framework of relations. They can be silent, ignored or overridden by other active objects, but they have developed enough solidity to move about and still hold their relations in place. For example, certain health and lifestyle behaviors, habits and/or conditions with solid relations to disease and risk of disease can eventually become immutable mobiles thus allowing a more generalized treatment of the pattern for groups of individuals/patients.

Obligatory Points of Passage: some immutable mobiles become obligatory points of passage, that is, central assemblages through which all relations in the network must flow at some time. This construct can be crucial for treating lifestyle and disease management, and more importantly for supporting clinical protocol alignment in cases of comorbidities.

In addition to this model and based on it we explore a system following the technologies and approaches described previously. More specifically, this work involves creat- 
ing a subset of the UMLS Semantic Network and introducing some more fields specific to comorbidities, in order to address principles pertaining to clinical pathways, including temporal properties and organization of entities. The ultimate goal is the development of an ontology to describe the model of comorbidities and their management. This ontology will be the basis for creating the social semantic heterogeneous network discussed in the previous section. Entity interactions within this network can be presented using graph visualization tools enabling further analysis and user exploratory navigation through the graph.

The proposed approach due to its nature of handling detailed patient data and other "sensitive" information has to take consideration of the exposure risks and apply some kind of anonymity that is counter-intuitive to the social aspect of a patient empowerment network. In order to circumvent that an anonymity layer should be introduced protecting the real identity of patients but guaranteeing the validity of the medical data and allowing the social interactions to be formed. A simple approach to that could be a frontend-backend compartmentalization of patient data. In the backend the system knows everything about a patient and has all the data available for the expert in order to manage the patient but in the frontend the patient is known only as a unique ID provided by the system. This allows patients to interact with the network without revealing anything they don't intend to.

\section{Discussion}

The co-existence of multiple networks of individuals, organizations and health conditions/interventions can be exploited in order to create different views of the healthcare environment, thus creating variable impact. For example, an individual-centered linking visualization enhances integrated personal management of healthcare, collaboration and expert finding services. On the other hand, an organization-based linking visualization supports administrative, strategy and financial oriented goals, at an institutional, national and international level. Finally, resource oriented linking visualization/organization may serve a variety of goals. For example, visualization based on health conditions and interventions places focus on epidemiology and generation of new evidence on a large scale.

Following the approach of a heterogeneous network to organize information objects and humans alike and record their variable interactions one can further exploit notions and concepts of the actor-network theory to analyze the social structures and thus eventually gain more insights on the organization and communication of information.

Acknowledgments. This work has been funded in part by the Project HELCOHOP: Development of Electronic Clinical Protocols, 2012-2015, MIS 375876, co-funded by European and National funds.

\section{References}

1. Lenz, R., Beyer, M., Kuhn, K.A.: Semantic integration in healthcare networks. Int. J Med. Inform. 76, 201-207 (2007)

2. Kodner, D.L., Spreeuwenberg, C.: Integrated care: meaning, logic, applications, and implications - a discussion paper. Int. Journal of Integrated Care 2(14) (November 2002) 
3. Gröne, O., Garcia-Barbero, M.: Trends in Integrated Care - Reflections on Conceptual Issues. World Health Organization, Copenhagen (2002)

4. Chatzimarkakis, J.: Why patients should be more empowered. J Diab. Science Technol. 4, 1570-1573 (2010)

5. Anderson, R.M., Funnell, M.M.: Patient empowerment: myths and misconceptions. Patient Educ. Couns. 79, 277-282 (2010)

6. van den Akker, M., et al.: Comorbidity or multimorbidity: what's in a name? Eur. J Gen. Pract. 2(2), 65-70 (1996)

7. Barnett, K., et al.: Epidemiology of multimorbidity and implications for health care, research, and medical education: a cross-sectional study. Lancet 380, 37-43 (2012)

8. Tinetti, M.E., et al.: Designing healthcare for the most common chronic condition: multimorbidity. JAMA 307, 2493 (2012)

9. Nagarajan, V., Tang, W.: Management of comorbid conditions in heart failure: a review. Med. Clin. North Am. 96(5), 975-985 (2012)

10. Valss, A., Gibert, K., Sanchez, D., Batet, M.: Using ontologies for structuring organizational knowledge in home care assistance. Int. J Med. Inform. 79, 370-387 (2010)

11. Knorr-Cetina, K.: Sociality with objects: social relations in postsocial knowledge societies. Theory, Culture \& Society 14(4), 1-30 (1997)

12. Engeström, J.: Why some social network services work and others don't. The case for object-centered sociality (2005), http: / / www. zengestrom.com/blog/2005/04/ why_some_social.html

13. Breslin, J., Decker, S.: The future of social networks on the Internet. The Need for Semantics. IEEE Internet Computing 11, 87-90 (2007)

14. Kaldoudi, E., Dovrolis, N., Dietze, S.: Information Organization on the Internet based on Heterogeneous Social Networks. In: ACM SIGDOC 2011: The 29th ACM International Conference on Design of Communication, Pisa, Italy, October 3-5, pp. 107-114 (2011)

15. Latour, B.: Reassembling the Social: An Introduction to Actor-Network Theory. Oxford University Press, Oxford (2005)

16. RDF, http://www.w3 . org/TR/rdf-syntax/

17. Bizer, C., Heath, T., Berners-Lee, T.: Linked Data - The story so far. IJSWIS 5, 1-22 (2009)

18. LOD,

http: / /www.w3 .org/wiki/SweoIG/TaskForces/CommunityProjects / L inkingOpenData

19. Gruber, T.R.: A translation approach to portable ontologies. Knowledge Acquisition 5(2), 199-220 (1993)

20. The National Center for Biomedical Ontology,

http: / /bioportal.bioontology.org/

21. Noy, N.F., Shah, N.H., Whetzel, P.L., Dai, B., Dorf, M., Griffith, N., Jonquet, C., Rubin, D.L., Storey, M.A., Chute, C.: BioPortal: ontologies and integrated data resources at the click of a mouse. Nucleic Acids. Res. 1, 37 (2009)

22. Bodenreider, O.: The Unified Medical Language System (UMLS): integrating biomedical terminology. Nucleic Acids Research 32, D267-D270 (2004)

23. Guthrie, B., et al.: Adapting clinical guidelines to take account of multimorbidity. BMJ 345, e6341 (2012)

24. National Library of Medicine, Chapter 5 - Semantic Networks. UMLS Reference Manual. U.S. National Library of Medicine, National Institutes of Health, Bethesda (2009) 